\title{
Relationship between functional movement screen scores and global positioning parameters associated with musculoskeletal injuries in soccer player
}

\author{
Serdar Arslan (1) Engin Dinç®i) 2 \\ ${ }^{1}$ Department of Physiotherapy and Rehabilitation, Faculty of Health Science, Necmettin Erbakan University, Konya, Turkiye. ${ }^{2}$ Konya
}

\begin{abstract}
The aim of this study was to investigate relationship Functional Movement Screening (FMS) scores and the Global Positioning Systems (GPS) data associated with musculoskeletal injuries soccer injuries in professional soccer players. The study included 19 soccer players with a mean age of $21.53 \pm 1.98$ years, a mean height of 180.58 $\pm 6.09 \mathrm{~cm}$, and a mean bodyweight of $73.08 \pm 5.29 \mathrm{~kg}$. The functional movement screening test battery was applied, and then GPS data associated with musculoskeletal injuries were recorded for each player during training sessions over a 6-week period. The recorded GPS data were mean total distance $5473.72 \pm 344.49 \mathrm{~m}$, mean distance per minute $69.11 \pm 6.59 \mathrm{~m}$, high-intensity running distance (14-20 km / h-1) $150.47 \pm 46.92 \mathrm{~m}$, very high intensity running distance ( $>20 \mathrm{~km} / \mathrm{h}-1) 34.94 \pm 16.96 \mathrm{~m}$, and body load of $108.43 \pm 15.87$. The GPS data of the participants with the FMS composite score above the cutoff point score and those below the cutoff point score were similar $(0.05<p)$. The composite scores of the participants who were above and below the cut-off point of the composite FMS score calculated according to the injury history were different $(\mathrm{p}<0.05)$. A two-way positive significant correlation was determined between deep squat, movement and composite FMS scores with VHIRD $(>20 \mathrm{~km} / \mathrm{h}$ 1). The scores obtained from the FMS test battery were not determined to affect the GPS data associated with musculoskeletal injuries recorded during training and associated with injuries. However, a significant relationship was determined between FMS scores and the amount of very high speed activity which is associated with musculoskeletal injuries in soccer players. Injury history is a determinant of the FMS composite score cut-off point and should be calculated separately for each population studied.
\end{abstract}

Keywords. Football, professional, running.

\section{Introduction}

Soccer is a sport which is both aerobic and anaerobic as the movement patterns include sprinting, jumping, changes of direction, and tackling (Carling, 2010). The physiological demands of soccer result in serious internal and external loads on the players (Mccall et al., 2016). Monitoring these loads is important to follow the match and training performances of players (Bompa \& Haff, 2009). In addition, it has been claimed that injuries in soccer are associated with the body load and therefore tracking body load can contribute to the prevention of injuries (Ehrmann et al., 2016; Cross et al., 2016).
The use of GPS systems has recently increased for the tracking of body load in soccer. These systems allow external loads to be monitored regularly and objectively during training and matches (Ravé et al., 2020). GPS variables have been reported to be highly correlated with other methods used to measue workloads. For example, there is evidence that there is a strong relationship between total distance, distance at different training intensities and body load with rated perceived exertion (Casamichana et al., 2013).

Functional movement screening (FMS) consists of a series of tests in which motor control, mobility, and reflex core stabilization are evaluated together. The

$凶$ S. Arslan, e-mail: serdararslan@erbakan.edu.tr

Received: December 12, 2021 - Accepted: December 30, 2021 - Published: December 31, 2021

To Cite: Arslan, S., Dinç, E. (2021). Relationship between functional movement screen scores and global positioning parameters associated with musculoskeletal injuries in soccer player. Turk J Kinesiol, 7(4), 132-140. DOI: 10.31459/turkjkin.1031274 
purpose of the screening system is to detect movement asymmetry and dysfunctions (Cook, 2010). It has been reported that there is a relationship between musculoskeletal system asymmetries and dysfunctions determined with this screening system and sports injuries and some performance parameters (Kraus et al., 2014; Parchmann \& Mcbride, 2011). Therefore, it has been reported that FMS scores can be used to predict performance and the injuries that an athlete may incur (Kraus et al., 2014; Parchmann \& Mcbride, 2011). There is also evidence that FMS scores in soccer are associated with lower extremity strength asymmetries (Sannicandro et al., 2017) and are successful in predicting injuries (Kiesel et al., 2007). In addition, exercise programs which have been developed to correct asymmetry and dysfunctions based on FMS scores have been shown to be effective in optimizing performance and preventing injuries (Campa et al., 2019).

The conditions under which a soccer match is played, the position of the player in the game, the level of competition, environmental factors, and match strategy and tactics affect the physical performance and body load of elite players (Carling et al., 2011; Gréhaigne et al., 2012). In addition, it is likely that there is a relationship between body load and individual physical features such as muscle strength, anatomical alignment disorders, and neuromuscular control (Hrysomallis, 2011). However, the relationship between these physical properties and body load is unclear. When it is considered that both FMS and body load are related to injuries, it is possible that there is a mutual correlation, but this has not been investigated as yet.

Knowledge of the relationship between GPS parameters related to soccer injuries and FMS will provide information about the effect of individual physical properties on body load. The hypothesis of this study was that the body load related to soccer injuries might be predicted by the FMS scores and that soccer players with lower FMS scores would have a higher body load related to soccer injuries than those with higher FMS scores. Therefore, the aim of this study was to examine the relationship between the two tools used in injury prediction and to determine whether FMS scores correlate with the GPS data related to soccer injuries in professional soccer players.

\section{Methods}

\section{Participants}

The study utilized a crossectional design to determine the relationship between FMS score and GPS data related to soccer injuries in professional soccer players. The study included 21 professional soccer players who were playing in teams in the 2nd Division of the Turkish Football Federation League. Exclusion criteria of test battery were as follows; having any musculoskeletal injury at the time of testing, having conducted high-intensity training within 24 hours prior to the application of the test protocol, verbally reported fatigue, having consumed alcohol within 48 hours prior to the application of the test protocol, having taken antiinflammatory/muscle relaxant / pain medication within 48 hours prior to the application of the test protocol, having consumed a stimulant substance like caffeine within 12 hours prior to the application of the test protocol (Marques et al, 2017), currently undertaking corrective exercise and unavailability of physiological load data in training sessions for any reason during the time period in which GPS data were collected.

\section{Procedure and Data Collection}

Participants were informed about the study. The demographic data of the participants were recorded, and conformity with the criteria for recruitment was assessed. The injury history of the participants who met the recruitment criteria was recorded. The FMS test battery was applied, after which GPS data were recorded in each training session for a period of 6 weeks.

Data about the injury history was collected by questionnaire. The injuries that occurred during soccer training or matches, caused musculoskeletal complaints were defined as soccer injuries (Fuller et al., 2006). Soccer injuries experienced by the participants during their soccer professional careers were recorded.

Participants were evaluated according to the FMS test guidelines. The FMS tests were applied using the official FMS test kit (Functional Movement Systems, Lynchburg, VA, USA). Each test explained and allowed the participants to perform the tests 3 times. After recording the data of the participants for the FMS test battery, the physiological load data during training started to be collected using the club's own GPS athlete tracking system (GPSports, Canberra Australia). The total distance (TD), per minute 
distance (PMD), high intensity running distance (HIRD) (14-20 km / h-1), very high intensity running distance (VHIRD) ( $>20 \mathrm{~km} / \mathrm{h}-1$ ), and body load (BL) data were recorded (Ehrmann et al., 2016). The average of the data recorded in each training session over a period of 6 weeks was calculated and used in the analysis.

\section{FMS}

FMS consists of a series of tests which can be easily applied in a short time and do not require complex measuring equipment. FMS tests can demonstrate functional movement deficits and asymmetries related to neuromuscular control, reflex core stabilization, and range of motion (Cook, 2010).

The test battery consists of seven movement patterns, which are scored between 0 and 3 based on observation. A score of 0 and 1 means that the test failed, while a score of 2 and 3 indicates that the test was successful. A test score of 0 is given if the tests cannot be performed due to pain, 1 if it cannot be performed in facilitated positions or even with compensation, 2 if it can be performed with a facilitated position or compensation, and 3 if it can be performed without compensation in the desired position (Cook et al., 2006, Cook et al., 2006).

The tests that make up the test battery include deep squat, hurdle step, inline lunge, shoulder mobility, activated straight leg raise, trunk stability push-up, and rotatory stability. Also, the protocol includes 3 clearing tests for shoulder mobility, trunk stability push-up, and rotatory stability tests. Being positive for these clearing tests indicates pathology that prevents the tests from being performed. The right and left sides are scored separately for five of these tests (hurdle step, inline lunge, shoulder mobility, activated straight leg raise, rotator stability). The lowest of the scores of the two sides is used as the test score. The composite FMS score is the total of the test scores of the seven movement patterns (Gray Cook et al., 2014, Gray Cook et al., 2014). The composite score is used to predict injuries. Different cutoff points have been reported for the prediction of injuries (Letafatkar et al.,2014; Kiesel et al., 2007). The total of the deep squat, hurdle step and inline lunge scores is evaluated as the movement score, the total of the shoulder mobility and active straight leg raise scores as the mobility score and the total of the trunk stability push-up and rotatory stability scores as the core stabilization score. Scores of $\geq 6$ for movement, $\geq 4$ for mobility and $\geq 4$ for core stabilization are accepted as threshold points. Thus, the screening system can be examined and interpreted under three main headings (Cook, 2010; Cook et al., 2006; Gray Cook et al., 2014). The FMS test battery has been reported to have high intratester and intertester test reliability, but there are doubts about its validity (Smith \& Hanlon, 2017).

\section{GPS}

GPS has been used in team sports since 2003 and has become the routine external training load measurement method, especially in soccer (Wehbe et al., 2014). GPS is a navigation system that calculates geographical location using information sent by more than one satellite (Larsson, 2003). Modern GPS units are integrated with a tri-axial accelerometer, which measures acceleration in three planes to calculate the composite force vector size, known as G-force (Scott et al., 2016). Accelerometers can be used to measure the forces acting on the athlete, which are referred to as the body load. It can also be used to measure the effect of athletes on other objects and surfaces (Cummins et al., 2013). With recent technological developments, the GPS unit has become quite small and lighter (Gabbett et al., 2012). Athletes wear a custom-made vest, in which there is a pocket between the shoulder blades where the GPS unit is placed. The data recorded by this unit are exported with special software (Wisbey et al., 2010). With GPS and integrated systems, it is possible to access data related to many internal or external loads at the same time, such as the total distance, the distance or the number of efforts at different speed acceleration thresholds, speed, body load, cardiac responses, and running economy (Vanrenterghem et al., 2017). In team sports, GPS devices have been shown to provide sufficiently reliable and accurate workload data (Coutts \& Duffield, 2010).

\section{Data Analysis}

Participants were grouped according to the composite FMS score calculated based on the injury history of the participant. The cutoff point of the FMS composite score was determined using receiver operator characteristic (ROC) analysis. Participants with a composite FMS score below the cutoff point formed the Below Composite Score Group (BCSG), and those above the cutoff formed the Above Composite Score Group (ACSG). The participants were also grouped as the Injury Group (IG) and the Non-Injury Group (NIG) according to the reported injury history. Data obtained in the study were analyzed statistically using SPSS vn. 21.0 software (IBM Corporation, Armonk, NY, USA). Numerical data 
were reported as mean \pm standard deviation (SD) values, and categorical data as number (n) and percentage (\%). Statistics were calculated for descriptive data, each FMS test score, composite FMS score, and GPS data. The Mann-Whitney U-test was used to compare the groups in respect of FMS scores and GPS variables. Correlations between GPS data and FMS scores were determined using Spearman Correlation Analysis. A value of $\mathrm{p}<0.05$ was accepted as statistically significant.

\section{Results}

Of the 21 subjects initially recruited, 2 were excluded from the study with the application of the exclusion criteria. Thus the data were examined of 19 male professional soccer players with a mean age of 21.53 \pm 1.98 years, mean height of $180.58 \pm 6.09 \mathrm{~cm}$ and mean body weight of $73.08 \pm 5.29 \mathrm{~kg}$.
At least one musculoskeletal injury was reported in the injury history of $73.68 \%$ of the soccer players. The most commonly reported injury type was lower limb joint injury $(50.00 \%)$. The distribution of the injuries reported in the injury history is shown in Figure 1.

The composite FMS score cutoff point calculated according to the injury history was found to be 11.50 . The BCSG included subjects with a composite FMS score of $\leq 11$, and the ACSG, those with a composite FMS score of $>11$. The mean composite FMS score was $10.22 \pm 0.97$ for the BCSG, and $13.40 \pm 1.35$ for the ACSG. The difference between the FMS composite scores of the groups was statistically significant $(p<0.05)$. The FMS scores of the groups are shown in detail in Table 1.

At least one asymmetrical pattern was present in $91.67 \%$ of the total participants, with at least one lower limb asymmetric pattern in 57.89\%. The distribution of asymmetric patterns is shown in Figure 2.

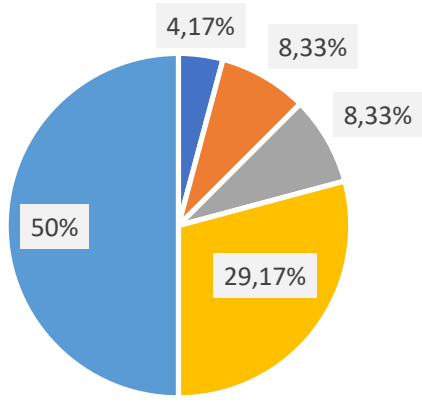

- Head injuries

Spine injuries

- Upper extremity muscle injuries

- Lower extremity muscle injuries

- Lower extremity joint injuries

Figure 1. The distribution of injuries reported by the participants in the injury history.

Table 1

FMS scores of the participants.

\begin{tabular}{|c|c|c|c|c|c|}
\hline \multirow{2}{*}{ Variables } & \multicolumn{2}{|c|}{ BCSG } & \multicolumn{2}{|c|}{ ACSG } & \multirow{2}{*}{$p^{a}$} \\
\hline & Median & $(\min -\max )$ & Median & $(\min -\max )$ & \\
\hline Deep squat & 1 & $1-2$ & 2 & $1-2$ & 0.118 \\
\hline Hurdle step & 2 & $2-2$ & 2 & $2-3$ & 0.343 \\
\hline Inline lunge & 2 & $1-2$ & 2 & $1-2$ & 0.098 \\
\hline Movement & 5 & $4-6$ & 6 & $4-7$ & $0.045^{*}$ \\
\hline Shoulder Mobility & 1 & $1-2$ & 2 & $1-2$ & 0.172 \\
\hline Active Straight leg raise & 2 & $1-2$ & 2 & $2-3$ & $0.026^{*}$ \\
\hline Mobility & 3 & $2-4$ & 4 & $3-6$ & $0.020^{*}$ \\
\hline Trunk stability push-up & 1 & $1-2$ & 2 & $2-3$ & $<0.001^{*}$ \\
\hline Rotatory stability & 1 & $1-2$ & 1 & $1-2$ & 0.165 \\
\hline Core Stability & 2 & $2-3$ & 3.5 & $3-5$ & $0.001^{*}$ \\
\hline Composite score & 11 & $9-11$ & 13.5 & $12-15$ & $<0.001^{*}$ \\
\hline
\end{tabular}

${ }^{a}$ Mann-Whitney U-test, BCSG: Below Composite Score Group, ACSG: Above Composite Score Group, *p<0.05 


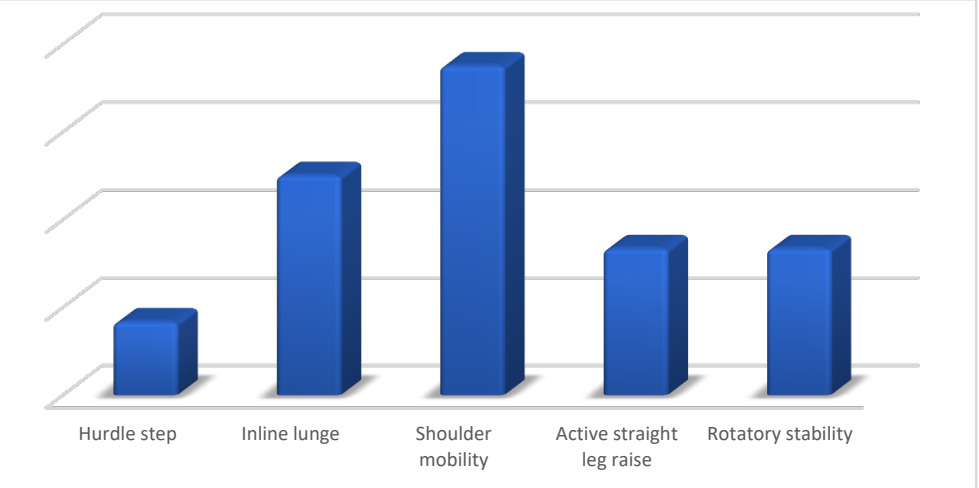

Figure 2. Distribution of asymmetric patterns.

Table 2

GPS data of the participants according to the FMS composite score and injury history.

\begin{tabular}{|c|c|c|c|c|c|c|}
\hline \multirow[b]{3}{*}{ Variables } & \multicolumn{3}{|c|}{ According to the FMS composite score } & \multicolumn{3}{|c|}{ According to the injury history } \\
\hline & BCSG & ACSG & & IG & NIG & \\
\hline & $\begin{array}{c}\text { Median } \\
\text { (min-max) }\end{array}$ & $\begin{array}{c}\text { Median } \\
\text { (min-max) }\end{array}$ & $\mathrm{p}^{\mathrm{a}}$ & $\begin{array}{l}\text { Median } \\
\text { (min-max) }\end{array}$ & $\begin{array}{l}\text { Median } \\
\text { (min-max) }\end{array}$ & $\mathrm{p}^{\mathrm{a}}$ \\
\hline Total distance & $\begin{array}{c}5420.44 \\
(5163.99-5755.73)\end{array}$ & $\begin{array}{c}5460.32 \\
(4990.88-6540.99)\end{array}$ & 0.744 & $\begin{array}{c}5400.75 \\
(5183.48-5755.73)\end{array}$ & $\begin{array}{c}5424.33 \\
(4990.88-6540.99)\end{array}$ & 1 \\
\hline Per minute distance & $\begin{array}{c}66.45 \\
(62.85-73.06)\end{array}$ & $\begin{array}{c}66.77 \\
(63.01-92.75)\end{array}$ & 0.744 & $\begin{array}{c}65.86 \\
(62.85-71.99)\end{array}$ & $\begin{array}{c}66.77 \\
(63.01-92.75)\end{array}$ & 0.355 \\
\hline $\begin{array}{l}\text { High intensity } \\
\text { running distance }\end{array}$ & $\begin{array}{c}120.13 \\
(72.29-213.87)\end{array}$ & $\begin{array}{c}159.51 \\
(101.88-266.56)\end{array}$ & 0.142 & $\begin{array}{c}163.82 \\
(72.29-213.87)\end{array}$ & $\begin{array}{c}134.88 \\
(101.89-266.56)\end{array}$ & 0.926 \\
\hline $\begin{array}{l}\text { Very high intensity } \\
\text { running distance }\end{array}$ & $\begin{array}{c}20.26 \\
(12.47-69.72)\end{array}$ & $\begin{array}{c}42.56 \\
(12.67-57.27)\end{array}$ & 0.050 & $\begin{array}{c}34.35 \\
(20.26-69.72)\end{array}$ & $\begin{array}{c}31.05 \\
(12.47-57.27)\end{array}$ & 0.459 \\
\hline $\begin{array}{l}\text { New total body } \\
\text { load }\end{array}$ & $\begin{array}{c}97.03 \\
(85.77-128.87)\end{array}$ & $\begin{array}{c}114.18 \\
(85.98-139.43)\end{array}$ & 0.142 & $\begin{array}{c}112.50 \\
(94.10-139.43)\end{array}$ & $\begin{array}{c}107.71 \\
(85.77-131.42)\end{array}$ & 0.139 \\
\hline
\end{tabular}

\begin{tabular}{lcc}
\multicolumn{2}{l}{$\begin{array}{l}\text { Table } 3 \\
\text { Relationships between FMS scores and very high intensity running distance. }\end{array}$} \\
\hline \multirow{2}{*}{ Variables } & \multicolumn{1}{c}{ Very high intensity running distance } \\
\cline { 2 - 3 } & Correlation Coefficient & $p^{\text {a }}$ \\
\hline Deep squat & 0.458 & $0.049^{*}$ \\
Hurdle step & 0.344 & 0.149 \\
Inline lunge & 0.349 & 0.143 \\
Movement & 0.510 & $0.026^{*}$ \\
Shoulder Mobility & 0.077 & 0.753 \\
Active Straight leg raise & 0.374 & 0.115 \\
Mobility & 0.230 & 0.344 \\
Trunk stability push-up & 0.412 & 0.080 \\
Rotatory stability & 0.044 & 0.859 \\
Core Stability & 0.304 & 0.205 \\
Composite & 0.490 & $0.033^{*}$
\end{tabular}


The mean value of TD was $5473.72 \pm 344.49 \mathrm{~m}$, PMD $69.11 \pm 6.59 \mathrm{~m}$, HIRD $150.47 \pm 46.92 \mathrm{~m}$, VHIRD $34.94 \pm 16.96 \mathrm{~m}$ and BL $108.43 \pm 15.87$ arbitrary units (a.u.). The difference between the GPS data of the BCSG and ACSG was not statistically significant. The difference between the GPS data of the IG and NIG was not statistically significant. The GPS data of the groups are shown in Table 2.

No correlation was determined between the FMS scores and TD, PMD, HIRD (14-20 km/h-1) and BL. A two-way positive significant correlation was deterrmined between deep squat, movement and composite FMS scores with VHIRD ( $>20 \mathrm{~km} / \mathrm{h}-1)$. The relationships between the FMS scores and VHIRD are shown in Table 3.

\section{Discussion}

The aim of this study was to examine the relationship between FMS scores and GPS data related to soccer injuries in professional soccer players. The results of the study demonstrated that injury-related data were not predicted by FMS scores calculated based on injury history. However, a two-way positive significant relationship was determined between FMS scores and VHIR.

Previous studies in literature have provided evidence that athletic injuries can be predicted by the FMS composite score (Kiesel et al., 2007; Chorba et al., 2010; Letafatkar et al., 2014). The widely accepted opinion is that athletes with a composite score of $<14$ from the FMS test battery are at greater risk of injuries (Kiesel et al., 2007; Chorba et al., 2010). However, different values have been reported for the FMS composite score cutoff-point for the prediction of injuries in athletes. Letafkter et al. reported that soccer players with a score of $<17$ are at 4.7 -fold greater risk of injury than those who score $>17$ (Letafatkar et al., 2014). According to the results of the current study, the FMS composite score cutoff point obtained based on the injury history was 11.50 . The discrepancies in cutoff points reported in previous studies could be attributed to differences in evaluators and the characteristics of the studied populations.

Soccer is a sport that requires a high level of physical exertion, and it has been shown that an elite male soccer player has to run between 9000-12000 meters in a single soccer match (Carling, 2010). Between $75 \%$ and $85 \%$ of this distance are runs that require aerobic effort at low speeds. The remaining part consists of anaerobic activities that require high physical effort, such as sprinting, jumping, acceleration, and deceleration (Bradley et al., 2009). The quality and quantity of anaerobic efforts is the most important determinant of the performance of an individual and the whole team (Reilly et al., 2000). The physical requirements of match and training are different as the distance covered and the amount of high-intensity activity in a match is greater than in training (De Silva et al., 2018). However, there are few studies that have analyzed GPS data related to training (Stevens et al., 2017). In a study examining GPS data of training sessions, Ehrmann FE et al reported TD of approximately $7 \mathrm{~km}$, HIRD of $800 \mathrm{~m}$, VHIRD of $275 \mathrm{~m}$, PMD of approximately $87.50 \mathrm{~m}$ and BL of 140 a.u. (Ehrmann et al., 2016). Rossi, A. et al. reported the TD as $3882.94 \pm 1633.21$ and the VHIRD as $410.67 \pm 221.29$ in a study, where an algorithm was presented to evaluate and interpret the relationships between injury risk and training performance in professional soccer (Rossi et al., 2018). In the current study, the mean TD was $5473.72 \pm 344.49 \mathrm{~m}$, PMD $69.11 \pm 6.59 \mathrm{~m}$, HIRD $150.47 \pm 46.92 \mathrm{~m}$, VHIRD $34.94 \pm 16.96 \mathrm{~m}$ and $\mathrm{BL}$ $108.43 \pm 15.87$ a.u.. In terms of GPS data, it is not possible to obtain standard values in every study due to different study methodologies, individual differences between participants, differences in competition level and variability of training content.

The relationship between FMS scores and athletic performance remains a topic of current interest. In this context, the effect of FMS scores on high-speed activity performance such as sprinting is an important issue that has been previously investigated. Parchmann, C. J. Studied golfers to determine whether 1 repetition of maximum strength squatting and FMS were associated with jumping, sprinting, agility skills and a skill specific to the sport. The results of that study demonstrated no relationship between FMS scores and $10 \mathrm{~m}$ and $20 \mathrm{~m}$ sprint times (Parchmann et al., 2011). Campa et al. examined the relationship between anthropometry, FMS and physical performance characteristics with repetitive sprinting ability in young male soccer players. The 40-meter repetitive sprint mean time was determined to be correlated with the FMS movement sub-score, and the best time of the 40meter repetitive sprint was correlated with the FMS composite score and the FMS movement sub-score. According to the same study results, the FMS composite score is a useful tool for predicting repetitive sprint performance (Campa et al., 2019). Koźlenia et al. investigated the relationship between movement quality with sprint and agility performance. Researchers reported that the sprint and agility performances of athletes who scored $\geq 14$ composite scores on the FMS test were better than 
those who scored $14>$ composite scores on the FMS test. Accordingly, they concluded that increasing the quality of movement can improve agility and sprint performance (Koźlenia et al., 2020). In another study investigating sprint and agility performance with FMS scores; Lee et al. reported that FMS composite score and sprint and agility performance were inversely correlated. The researchers concluded that having a high FMS composite score would positively affect sprint and agility performance (Lee et al., 2019). The most important result of the current study, which has not been emphasized before in the literature, was that a relationship was determined between the FMS scores and the VHIRD recorded during the training period. The findings of the present study also showed a two-way positive correlation between deep squat, FMS movement subscore, and FMS composite score, and VHIRD.

There is also evidence that individual test scores give more information about performance than FMS composite score. Silva et al. reported that trunk stability push up test, which evaluates core stabilization, can be used as a predictor of physical performance (Silva et al., 2017). Campa et al. reported that as repetitive sprint performance includes changing direction, and depends on the synergistic interaction between motor control and core stability, this explains the relationship between repetitive sprint performance and FMS scores (Campa et al., 2019). In the current study, it was seen that the deep squat test and, accordingly, the motor control subcategory score were correlated with VHIRD. Results supported that the synergistic interaction between motor control and core stability also affects the VHIRD distance demonstrated during the training period.

This study had some limitations, primarily the use of the results of the FMS test battery applied only once with GPS data in a certain part of the season. In future studies, more explanatory information could be obtained by analyzing FMS scores repeated several times during the season and GPS data recorded throughout the season.

To the best of our knowledge, there is no research in the literature that has investigated the effect of FMS on GPS data. The results of this study can therefore be considered of value in providing important information to clarify this issue. The study results showed that the FMS composite score cutoff point calculated based on the injury history, and the injury history do not affect the GPS data associated with the injury. However, deep squat, FMS movement sub-score, and FMS composite score were seen to be correlated with VHIRD.

\section{Conclusion}

In conclusion, the FMS test battery provides important information about the musculoskeletal asymmetry and dysfunction of male soccer players. However, the scores obtained from the FMS test battery do not affect the GPS data recorded during training and which have been reported to be associated with injuries. However, FMS composit score and deep squad test score are coraleted with the amount of very high speed activity. The amount of very high speed activity in the training and games may be increased with movement quality with programs that will increase the quality of movement, especially deep squad pattern in soccer.

In the future, there is a need for studies investigating the effect of approaches that increase the quality of movement on the amount of very high speed activity. In addition, examining the relationship between football injuries which occur during high speed and FMS scores also needs to be investigated.

\section{Acknowledgments}

None.

\section{Ethical Considerations}

This cross-sectional study was approved by the Council on the Ethics of Non-Pharmaceutical and Non-Medical Research of Meram Faculty of Medicine, Necmettin Erbakan University (Approval Number: 2019/2021, Meeting Number: 100, Date: 27/12/2019).

\section{Conflict of Interests}

We declared no conflict of interests regarding the publication of this manuscript.

\section{Funding}

This research did not support by specific grant from funding agencies in public, commercial, or not-forprofit sectors.

\section{References}

Bompa, T. O., \& Haff, G. G. (2009). Periodization. Theory and methodology of training. Campaign, IL: Human Kinetics. 
Bradley, P. S., Sheldon, W., Wooster, B., Olsen, P., Boanas, P., \& Krustrup, P. (2009). High-intensity running in English FA Premier League soccer matches. J Sports Sci, 27(2), 159-168.

Campa, F., Semprini, G., Júdice, P. B., Messina, G., \& Toselli, S. (2019). Anthropometry, physical and movement features, and repeated-sprint ability in soccer players. Int J Sports Med, 40(02), 100-109.

Campa, F., Spiga, F., \& Toselli, S. (2019). The effect of a 20week corrective exercise program on functional movement patterns in youth elite male soccer players. $J$ Sport Rehabil, 28(7), 746-751.

Carling, C. (2010). Analysis of physical activity profiles when running with the ball in a professional soccer team. J Sports Sci, 28(3), 319-326.

Carling, C., Dupont, G., \& Le Gall, F. (2011). The effect of a cold environment on physical activity profiles in elite soccer match-play. Int J Sports Med, 32(07), 542-545.

Casamichana, D., Castellano, J., Calleja-gonzalez, J., San román, J., \& Castagna, C. (2013). Relationship between indicators of training load in soccer players. J Strength Cond Res, 27(2), 369-374.

Chorba, R. S., Chorba, D. J., Bouillon, L. E., Overmyer, C. A., \& Landis, J. A. (2010). Use of a functional movement screening tool to determine injury risk in female collegiate athletes. N Am J Sports Phys Ther, 5(2), 47.

Cook G., Burton L., Hoogenboom, B. J., \& Voight, M. (2014). Functional movement screening: the use of fundamental movements as an assessment of functionpart1. Int J Sports Phys Ther, 9(3), 396.

Cook G., Burton L., Hoogenboom, B. J., \& Voight, M. (2014). Functional movement screening: the use of fundamental movements as an assessment of functionpart 2. Int J Sports Phys Ther, 9(4), 549.

Cook, G. (2010). Movement: Functional movement systems: Screening, assessment. Corrective Strategies (1st ed.). Aptos, CA: On Target Publications, 73-106.

Cook, G., Burton, L., \& Hoogenboom, B. (2006). Preparticipation screening: the use of fundamental movements as an assessment of function-part 1. $\mathrm{N} \mathrm{Am} \mathrm{J}$ Sports Phys Ther, 1(2), 62-72.

Cook, G., Burton, L., \& Hoogenboom, B. (2006). Preparticipation screening: the use of fundamental movements as an assessment of function-part 2. $\mathrm{N} \mathrm{Am} \mathrm{J}$ Sports Phys Ther, 1(3), 132-139.

Coutts, A. J., \& Duffield, R. (2010). Validity and reliability of GPS devices for measuring movement demands of team sports. J Sci Med Sport, 13(1), 133-135.

Cross, M. J., Williams, S., Trewartha, G., Kemp, S. P., \& Stokes, K. A. (2016). The influence of in-season training loads on injury risk in professional rugby union. Int $J$ Sports Physiol Perform, 11(3), 350-355.

Cummins, C., Orr, R., O’Connor, H., \& West, C. (2013). Global positioning systems (GPS) and microtechnology sensors in team sports: a systematic review. Sports Med, 43(10), 1025-1042.

De Silva, V., Caine, M., Skinner, J., Dogan, S., Kondoz, A., Peter, T., Axtell, E., Birnie, M., \& Smith, B. (2018). Player Tracking Data Analytics as a Tool for Physical Performance Management in Football: A Case Study from Chelsea Football Club Academy. Sports (Basel, Switzerland), 6(4), 130.

Ehrmann, F. E., Duncan, C. S., Sindhusake, D., Franzsen, W. N., \& Greene, D. A. (2016). GPS and injury prevention in professional soccer.J Strength Cond Res, 30(2), 360367.

Fuller, C. W., Ekstrand, J., Junge, A., Andersen, T. E., Bahr, R., Dvorak, J., Hagglund, M., McCrory, P., \& Meeuwisse, W. H. (2006). Consensus statement on injury definitions and data collection procedures in studies of football (soccer) injuries. Scand J Med \& Sci Sports, 16(2), 83-92.

Gabbett, T. J., Jenkins, D. G., \& Abernethy, B. (2012). Physical demands of professional rugby league training and competition using microtechnology.J Sci Med Sport, 15(1), 80-86.

Gréhaigne, J. F., Richard, J. F., \& Griffin, L. L. (2012). Teaching and learning team sports and games. Routledge.

Hrysomallis, C. (2011). Balance ability and athletic performance. Sports Med, 41(3), 221-232.

Kiesel, K., Plisky, P. J., \& Voight, M. L. (2007). Can serious injury in professional football be predicted by a preseason functional movement screen?. N Am J Sports Phys Ther, 2(3), 147.

Koźlenia, D., Domaradzki, J., Trojanowska, I., \& Czermak, P. (2020). Association between speed and agility abilities with movement patterns quality in team sports players. Med. Dello Sport, 73, 176-186.

Kraus, K., Schütz, E., Taylor, W. R., \& Doyscher, R. (2014). Efficacy of the functional movement screen: a review.J Strength Cond Res, 28(12), 3571-3584.

Larsson, P. (2003). Global positioning system and sportspecific testing. Sports Med, 33(15), 1093-1101.

Lee, S., Kim, H., \& Kim, J. (2019). The Functional Movement Screen total score and physical performance in elite male collegiate soccer players. J Exerc Rehabil, 15(5), 657.

Letafatkar, A., Hadadnezhad, M., Shojaedin, S., \& Mohamadi, E. (2014). Relationship between functional movement screening score and history of injury. Int $J$ Sports Phys Ther, 9(1), 21.

Marques, V. B., Medeiros, T. M., De Souza Stigger, F., Nakamura, F. Y., \& Baroni, B. M. (2017). The Functional Movement Screen (FMS ${ }^{\mathrm{TM}}$ ) in elite young soccer players between 14 and 20 years: Composite score, individualtest scores and asymmetries. Int $J$ Sports Phys Ther, 12(6), 977. 
Mccall, A., Dupont, G., \& Ekstrand, J. (2016). Injury prevention strategies, coach compliance and player adherence of 33 of the UEFA Elite Club Injury Study teams: a survey of teams' head medical officers. $\mathrm{Br} J$ Sports Med, 50(12), 725-730.

Parchmann, C. J., \& Mcbride, J. M. (2011). Relationship between functional movement screen and athletic performance. J Strength Cond Res, 25(12), 3378-3384.

Ravé, G., Granacher, U., Boullosa, D., Hackney, A. C., \& Zouhal, H. (2020). How to Use Global Positioning Systems (GPS) Data to Monitor Training Load in the "Real World" of Elite Soccer. Front Physiol, 11, 944.

Reilly, T., Bangsbo, J., \& Franks, A. (2000). Anthropometric and physiological predispositions for elite soccer. $J$ Sports Sci, 18(9), 669-683.

Rossi, A., Pappalardo, L., Cintia, P., Iaia, F. M., Fernández, J., \& Medina, D. (2018). Effective injury forecasting in soccer with GPS training data and machine learning. PLoS One, 13(7), e0201264.

Sannicandro, I., Cofano, G., Rosa, A. R., Traficante, P., \& Piccinno, A. (2017). Functional movement screen and lower limb strength asymmetry in professional soccer players. Br J Sports Med, 51(4), 381-382.

Silva, B., Clemente, F. M., \& Martins, F. M. (2017). Associations between functional movement screen scores and performance variables in surf athletes. J Sports Med Phys Fitness, 58(5), 583-590.
Scott, M. T., Scott, T. J., \& Kelly, V. G. (2016). The validity and reliability of global positioning systems in team sport: a brief review. J Strength Cond Res, 30(5), 1470-1490.

Smith, P. D., \& Hanlon, M. P. (2017). Assessing the effectiveness of the functional movement screen in predicting noncontact injury rates in soccer players. $J$ Strength Cond Res, 31(12), 3327-3332.

Stevens, T. G., De Ruiter, C. J., Twisk, J. W., Savelsbergh, G. J., \& Beek, P. J. (2017). Quantification of in-season training load relative to match load in professional Dutch Eredivisie football players. Sci Med Footb, 1(2), 117125.

Vanrenterghem, J., Nedergaard, N. J., Robinson, M. A., \& Drust, B. (2017). Training load monitoring in team sports: a novel framework separating physiological and biomechanical load-adaptation pathways. Sports Med, 47(11), 2135-2142.

Wehbe, G. M., Hartwig, T. B., \& Duncan, C. S. (2014). Movement analysis of Australian national league soccer players using global positioning system technology.J Strength Cond Res, 28(3), 834-842.

Wisbey, B., Montgomery, P. G., Pyne, D. B., \& Rattray, B. (2010). Quantifying movement demands of AFL football using GPS tracking. J Sci Med Sport, 13(5). doi: 10.1016/j.jsams.2009.09.002. 